\title{
Nanoscale toughening mechanism of nacre tablet
}

\author{
Ning Zhang ${ }^{1 *}$, Shengfeng Yang ${ }^{2}$, Liming Xiong ${ }^{3}$, Yu Hong ${ }^{4}$ and Youping Chen ${ }^{4}$ \\ ${ }^{1}$ Department of Civil and Environmental Engineering, Rice University, Houston, TX, 77005, US \\ ${ }^{2}$ Department of Material Science, University of California, San Diego, CA, 92093, US \\ ${ }^{3}$ Department of Aerospace Engineering, Iowa State University, Ames, IA, 50011, US \\ ${ }^{4}$ Department of Mechanical and Aerospace Engineering, University of Florida, Gainesville, FL, 32611, US
}

\begin{abstract}
Nacre has attracted widespread interest because its unique hierarchical structure, which is assembled by $95 \mathrm{wt} \%$ brittle aragonite and $5 \mathrm{wt} \%$ soft organic materials, leads to several orders of improvement in fracture toughness. Apart from the well proposed toughening mechanisms such as mineral bridges and tablets interlocks, the organic materials including biopolymers between tablets and proteins exist within a tablet can also potentially improve the toughness. In this work, we employ a novel approach combining steered molecular dynamics (SMD) and classical molecular dynamics (MD) to build a model of mineralprotein composite to mimic nacre tablet. The critical role of protein in improving the fracture toughness of nacre is investigated for the first time. MD simulations of single crystalline aragonite, polycrystalline aragonite and mineral-protein composite under uniaxial tensile loading are performed, and the obtained constitutive responses are compared with experimental measurements of nacre under tension. It is shown that the fracture toughness of mineral-protein composite is significantly larger than that of single crystalline or polycrystalline aragonite. Detailed atomic configuration analyses reveal that the fracture of individual computer model is governed by its unique failure mechanisms. Dislocation motion and phase transformation are observed during the failure of single crystalline aragonite. Polycrystalline aragonite fails by the inter-granular cleavage, as well as phase transformation within grain. It is surprisingly noted that other than the stretching of protein chains on grain boundaries, intra-granular fracture is triggered in mineral-protein composites. Proteins serve as strong glue between the inorganic nanograins. It is believed that the strong electrostatic interaction between protein and aragonite nanograins, combined with the remarkable plastic ductility of protein lead to the intra-granular failure, which consequently enhance the fracture toughness of the whole specimen.
\end{abstract}

\section{Keywords:}

Nacre tablet; Toughening mechanism; Protein-mineral interaction; Molecular dynamics; Aragonite; Electrostatic interaction ${ }^{1}$

\section{Introduction}

Nacre is the inner iridescent layer of mollusk shell, as shown in Fig. 1a [1]. The structure of nacre has been studied over decades owing to its remarkable mechanical performance. As in most structural biomaterials, nacre has a very complex hierarchical structure that spans over multiple length scales from

\footnotetext{
* Corresponding author, Tel.: 352-871-4498; Fax: 352-392-7303

E-mail: ningzhang@ufl.edu
} 
nanoscale to macroscale (Fig. 1b-f [1]). Nacre is made up of $95 \mathrm{wt} \%$ brittle aragonite (Fig. 1f), which is a crystallographic form of calcium carbonate $\left(\mathrm{CaCO}_{3}\right)$, and $5 \mathrm{wt} \%$ organic materials primarily that composed of proteins (Fig. 1g) and polysaccharides [2]. Surprisingly, the fracture toughness of nacre is about 3000 times higher than that of its major constituent - pure aragonite [3, 4], which is incomparable to conventional synthetic materials.

At the microscale, numerous mechanical experiments have been carried out with the attempt to improve the understanding of the relationship between hierarchical structure and remarkable mechanical property of nacre. The previous research of revealing toughening mechanisms of nacre has mainly focused on the structure of laminates [5, 6]. One of the key mechanisms proposed was the tablet sliding [7], which was controlled by the structure of interface between tablets such as asperities [8] and mineral bridges [9], as well as the waved surfaces of tablet [10] and interlocks between adjacent tablets [11]. However, unfortunately, these proposed structural mechanisms cannot comprehensively elucidate the dramatic enhancement of toughness in nacre. Actually, the hierarchical structure of nacre requires analyzing the mechanisms at the various scales.

Thanks to scanning electron microscope (SEM), the hard aragonite tablets were found to be glued together with soft biopolymers to form the brick-and-mortar structure [12-14]. Chitin fibers were reported to be the structural components of the intertile organic material [1]. Considerable progresses have been achieved in understanding the features of grain boundaries and the role of soft organic matrix in nacre. It was found that despite the small percentage of organic material in nacre, the polymer and protein matrices played an important role in crystal nucleation and growth [15, 16]. Although Meyers et al. [12] claimed that organic layers had a minor role in improving mechanical strength of nacre, by using AFM Smith et al. [17] have demonstrated that the organic component contributed significant to the high energy dissipation and hence high fracture toughness of nacre. In addition, the biopolymer in nacre was observed to have the capability to strengthen itself during deforming [18], which will contribute to the ultrahigh toughness of nacre.

At the nanoscale, facilitated by high-resolution transmission electron microscopy (TEM) the individual tablet was found to be not single crystalline aragonite but is, in turn, assembled by polygonal nanograins of about 3-10nm in size with proteins spacing in between nanograins [13, 19], which provides a ductile nature to such hierarchical structure [20]. Great efforts have been undertaken experimentally in identifying the sequences of various proteins, for instance, lustrin A [21], perlucin [22], perlustrin [23] and perlwapin [24]. Accordingly, based on the observation of in situ atomic force microscopy (AFM), nanograin rotation and deformation along with the lubrication of soft organic matrix on grain boundaries were proposed to make a significant contribution to the energy dissipation in nacre $[25,26]$. As a suitable tool to address the interaction from atomistic scale, steered molecular dynamics (SMD) simulations have been conducted to investigate the interaction between aragonite nanograin and proteins [27]. Different from the widely believed protein self-unfolding mechanism, the electrostatic interaction between protein and mineral was found to be responsible for the saw-tooth behavior observed from AFM experiment [17]. 
In this work, to systematically reveal the toughening mechanism of nacre on nanoscale, a model of mineral-protein composite is constructed, as shown in Fig. 1e. It is well known that grain boundaries play an important role in the mechanical response of polycrystalline materials. Various protein chains are introduced into the grain boundaries. We aim at identifying the critical role of protein plays in improving the toughness of nacre on nanoscale through computational modeling. It is worth emphasizing that the structure and component will be different for varying series of nacre. In the current work, we focus on the nacre from Red Abalone. A novel approach combining steered molecular dynamics (SMD) and classical molecular dynamics (MD) is employed. Since most of the experimental tests are conducted under hydration condition, herein water molecules are added on grain boundaries of mineral-protein composite to mimic the hydrated state of nacre. Uniaxial tensile loadings are applied to investigate the mechanical response. MD simulations of single crystalline and polycrystalline aragonite are also carried out to make comparisons with that of mineral-protein composites.

\section{Simulation Details}

\subsection{Computer models}

Molecular dynamics simulation has become more prevalent due to its capacity of building bottom-up models and revealing the plastic mechanisms, such as dislocation motion, phase transformation from atomic viewpoint $[28,29]$. In the current study, three computer models, i.e., single crystalline aragonite, polycrystalline aragonite and mineral-protein composite, are generated. The model of mineral-protein composite is constructed by adopting polycrystalline aragonite with proteins and water molecules intercalated in grain boundaries.

The unit cell structure of aragonite that used to build the computer models has been fully depicted in the work by Zhang and Chen [29]. Fig. 2a and Fig. 2b present the initial computer models of single crystalline and polycrystalline aragonite, which contain 1,739,200 and 1,687,250 atoms, respectively. The size of the computer models are $43.5 \times 43.5 \times 10.5 \mathrm{~nm}^{3}$. It is worthy to emphasis that in both models, the "caxis" of crystal aragonite aligns normal to the plane of the tiles (illustrated in Fig. 2a,b), which is consistent with the experimental observation [1]. For polycrystalline aragonite, the crystallographic orientation of individual grain is random within plane. The average size of nanograins is $10 \mathrm{~nm}$ in length, which is also in good agreement with the experimental measurement [13].

As for the model of mineral-protein composite, the size is $43.5 \times 43.5 \times 10.5 \mathrm{~nm}^{3}$. Four experimentally identified nacre proteins: lustrin A, perlucin, perlustrin and perwapin, the details of which were discussed in the work by Zhang and Chen [27], tangle together to represent the soft organic layer intercalated in grain boundaries. All of these proteins are isolated from the nacreous layer of the shell of abalone. Lustrin A is the first found large multi-domain protein that isolated from the nacreous layer of the abalone $H$. rufescens [21]. It is also the most complex in its primary structure and the most "popular" one of all the molluscan proteins. Lustrin A establishes a link between a primary structure and the overall mechanical property of nacre. Since the full-length of lustrin A is so long (4,439 bp), two typical domains, C1-P1-C2$\mathrm{P} 2$ and GS, are chosen to represent the structure of lustrin A in this work. Perlucin is an N-glycosylated 
protein with a character of calcium-dependent lectin (C-type) [22]. It was found that perlucin played an important role in biomineralization [30], consequently may have a strong influence on the mechanical properties of biominerals. Perlustrin is a small protein with an insulin-like growth factor binding $\mathrm{N}$ terminal domain, which may be biologically related [23]. Perlwapin contains 3 repeats of 40 amino acid residues with similarity to the whey acidic proteins (WAP) [24]. It is suggested that perlwapin inhibits the growth of certain crystallographic planes and could also play a role in the polymorph transition and mechanical functions.

Comparing with atomically ordered crystalline aragonite, proteins are highly disordered, which leads to the most challenging issue that how to determine the arrangements of protein chains on grain boundaries of mineral-protein composite. In this study, by means of general MD program AMBER, steered molecular dynamics (SMD) method with AMBER ff99 force field [31] is employed to build the model of mineral-protein composite. Actually, SMD is an increasingly popular tool for accelerating conformational changes in biomolecular system through the application of external forces. Unfortunately, since AMBER does not focus on large-scale molecular dynamic simulation, the maximum number of atoms in computer model that the AMBER can afford is 300,000, which is significantly smaller than our current model. Since only grain boundaries are used to constrain the position of protein chains during model construction, a hollow polycrystalline aragonite, as shown in Fig. 3, is generated by removing the atoms within nanograins. With this method, the total number of atoms drops from 1,600,000 to 216,150, which meets the requirement of AMBER program.

Fig. 3 illustrates our implementation of dragging one protein chain into grain boundaries. First, couples of reference points are predefined on grain boundaries and protein chain. For simplicity, only six couples of them, i.e., $A-A^{\prime}, B-B^{\prime}, C-C^{\prime}, D-D^{\prime}, E-E^{\prime}, F-F^{\prime}$, are presented in Fig. 3. The nanograin boundaries are fixed throughout the model construction. SMD simulation with constant velocity is performed to stretch the reference points on protein $\left(A^{\prime}, B^{\prime}, C^{\prime}, D^{\prime}, E^{\prime}, F^{\prime}\right)$ toward the corresponding ones on grain boundaries $(A$, $B, C, D, E, F)$. The movies 1_perlucin, 2_perwapin, 3_perlustrin and 4_lustrin A in the supplementary material display the top views of the process of dragging perlucin, perwapin, perlustrin and lustrin A into grain boundaries of polycrystalline aragonite. This procedure is very similar to pulling a protein chain out of a nacre tablet in AFM experiment [17], except that the pulling speed in computer simulation is of one hundred times higher than that in the experiment due to the limited time and length scales. The external force exposed on the reference points on protein chain can be expressed as,

$$
F=k(v t-x)
$$

where $x$ is the displacement of the reference atom on protein from its original position, $v$ is the pulling velocity, which is controlled at $1.0 \AA / \mathrm{ps}$ in our simulations, $v t$ is the position of the reference point on grain boundaries, and $k$ is the spring constant, which is controlled at $10.0 \mathrm{k}_{\mathrm{B}} \mathrm{T} / \AA^{2}$.

After all protein chains are dragged into grain boundaries, the previously removed nanograin atoms are returned to the original location, as shown in Fig. 4a. 28 protein chains including 63,000 atoms have been stretched into the grain boundaries. Therefore, the total number of atoms of the mineral-protein composite 
model is $1,663,000$, which far exceeds the affordable tolerance of AMBER. Hence, alternatively the massively paralleled program - LAMMPS [32] is employed to carry on the successive MD simulations.

Additionally, water is reported has a key effect on mechanical properties of nacre. Based on the experimental measurement, the tensile strength of nacre was $170 \mathrm{MPa}$ for dry and $140 \mathrm{MPa}$ for wet sample [4]. To simulate the hydrated nacre tablet, TIP3P water model [33] is employed and water molecules are randomly scattered around protein chains, as presented with red color in Fig. 4b,c.

\subsection{Interatomic potential}

It is a crucial step to choose an appropriate force field to account for the reaction between atoms in MD simulations. In the current work, the empirical potential developed by Dove et al. [34], which has been demonstrated can accurately predict the mechanical properties of aragonite [29], is adopted to take charge of the atomic interaction in aragonite.

The Dove potential function includes both bonded and non-bonded parts. For the bonded part, bondbending of O-C-O is described by harmonic function, which takes the form:

$V(\theta)=\frac{1}{2} k_{\theta}\left(\theta-\theta_{0}\right)^{2}$

where $k_{\theta}$ is the bond bending force constant, $\theta_{0}$ is the equilibrium bond angle and $\theta$ is the bond angle between the O-C-O atoms. An additional four-body term is used to model the planarity of the $\mathrm{CO}_{3}$ molecular ion. This torsional term is in the form:

$V(\varphi)=k_{t}[1-\cos (2 \varphi)]$

where $k_{t}$ is the out-of-plane force constant, $\varphi$ is the angle between two O-C-O planes in a single molecular ions.

For the non-bonded part, Coulomb interaction is evaluated using Ewald sum, with a precision of $1 \times 10^{6}$. The short-range interaction between $\mathrm{Ca}-\mathrm{O}, \mathrm{O}-\mathrm{O}$ and $\mathrm{C}-\mathrm{O}$ is treated using Born-Mayer repulsive potential, which takes the form:

$V(r)=A \exp (-r / \rho)$

where $A$ and $\rho$ are parameters particular to the types of atom interacting, with $r$ being the separation between two atoms. Full details of these parameters are tabulated in Table 1.

For proteins, AMBER ff99 force field [31] has been demonstrated to be appropriate for structural deformation including chains folding and unfolding [27]. The atomic interaction between the mineral and organic component is more complicated than that in inorganic crystals. In the current work, mineralprotein interaction is also modeled using the AMBER ff99 force field [31], which takes the form 
where $K_{b}, K_{\theta}, K_{\chi}$ and $K_{i m p}$ are the bond, angle, dihedral and improper force constants, respectively; b, $\theta, \chi$ and $\phi$ are the bond length, bond angle, dihedral angle and improper torsion angle, respectively, with the subscript zero denoting the equilibrium values for each term. $A_{i j}, B_{i j}$ are the distances at the Lennard-Jones minimum, $q_{i}, q_{j}$ are the partial atomic charges, and $\mathrm{r}_{\mathrm{ij}}$ is the distance between atoms $i$ and $j$.

Steered molecular dynamics (SMD) simulations are conducted using the AMBER simulation package [31]. Constant volume and temperature (NVT) molecular dynamics (MD) simulations are carried out with MD program LAMMPS [32]. As two different kinds of software, AMBER and LAMMPS will take different strategies to achieve the same goal. However, the format of force field and main algorithm employed in these two programs should be exactly the same. The force field as presented in Eq. 5 will be adopted in LAMMPS. After choosing the right format of force field, the coordinate and parameter topology can be transferred from AMBER to LAMMPS by using amber2lammps.py, which is provided by LAMMPS package. Moreover, in AMBER, bond lengths and angles involving all hydrogen atoms are constrained with the SHAKE algorithm, so that the model could be equilibrated. Therefore, in the molecular simulation by using LAMMPS, the computer model obtained by AMBER is adopted as the original model with SHAKE algorithm being applied on bonds in which hydrogen atoms are involved.

The Verlet-Leapfrog algorithm [35, 36] with a time step of $1 \mathrm{fs}$ is used to integrate the equations of motion. The Nosé-Hoover thermostat $[37,38]$ method with a relaxation constant of $0.1 \mathrm{ps}$ is used to control the temperature at a constant value $(298 \mathrm{~K})$. The non-bonded Coulomb and Van der Waals interactions are calculated with a cut-off of $15.0 \AA$.

\section{Results and discussion}

It should be noted from Fig. 4a,b that considerable spacing exists between protein and aragonite nanograins. To ensure proteins and aragonite are in close contact, compressive loadings along x- and yaxis are applied on the model in Fig. 4b to eliminate the spacing on grain boundaries. Afterward, the entire system is allowed to carry out full relaxation for a period of 1ns. Eventually, the average width of grain boundaries achieves the value of $1.2 \mathrm{~nm}$, which is consistent with experimental measurement [14].

Thereafter, uniaxial tensile loading along $\mathrm{x}$-axis is applied on the models of single crystalline aragonite, polycrystalline aragonite and mineral-protein composite, respectively. The strain rate is controlled at $2 \times 10^{7} / \mathrm{s}$. The obtained stress-strain relations are plotted in Fig. 5a. We also present the experiment results [20] in Fig. 5b for comparison, in which the work-of-fracture of hydrated nacre is significantly larger than that of pure aragonite or dry nacre. The samples of single crystalline aragonite, polycrystalline aragonite 
and mineral-protein composite in the simulations are adopted to mimic the pure aragonite, dry nacre and hydrated nacre in experiment. It can be clearly noted that although the three computer models shows distinct failure modes, the overall deformation patterns are similar to the observations from experiment [20]. It is worthy to emphasis that the computer model in MD simulation is perfect crystalline aragonite that has excluded the defects such as impurity and pores, which are inevitable in experiment. Secondly, the loading is controlled by displacement in our simulations instead of force control in experiment. Furthermore, the empirical force field employed in MD simulation is developed to mimic but cannot exactly reproduce the interactions between atoms. Another reason for the mismatch between predicted stress values and the experimental values is due to the high strain rate loadings employed in the present MD simulations since the timescale in MD is limited at picosecond level. However, the issue of inconsistent timescales between MD simulations and experiments (microseconds or seconds) has not yet been fully addressed by any classical MD simulations. Consequently, the magnitudes of stress and strain from simulations are larger than experimental measurements.

For single crystalline aragonite, at the initial elastic deformation stage, stress increases linearly with strain until reaches its fracture strength of 970MPa. The slope of stress-strain curve in this region gives the Young's modulus of $75 \mathrm{GPa}$, which is slightly smaller than the experimental estimation (87GPa) [20]. After the yielding point, the stress suddenly drops, which indicates the failure of specimen. To identify the underlying plastic deformation mechanism, in Fig. 6a,b we present the atomic configurations of single crystalline aragonite at strains of $\square=1.5 \%$ and $\square=2.0 \%$, respectively. The atoms are colored according to coordinate number with green for aragonite, blue for new phase and dislocation line. It is found that with the increase of tensile strain dislocations nucleate and propagate along $\{110\}$ slip planes. The dislocation leading partial and trailing partial have been marked in Fig. 6a. When the dislocation line along [110] direction meets the one along [-110] direction, a dislocation lock forms and the atomic structure of the area ahead of dislocation lock is changed, but still maintains a long-range crystalline order, which indicates the transformation of new phase, as shown in Fig. 6a,b.

As for the case of uniaxial tension of polycrystalline aragonite, it can be observed from Fig. 5a that the stress-strain curve is inelastic behavior from the very beginning. Compared to uniaxial tension of single crystalline aragonite, the fracture strength of polycrystalline aragonite is reduced to $300 \mathrm{MPa}$. The stress drops gently after yielding point. To identify the deformation mechanisms behind the stress-strain relation; we present the atomic arrangements of polycrystalline aragonite at strain $\square=3.0 \%$ in Fig. $7 \mathrm{a}$. Compared with the initial configuration in Fig. 2b, it is noted that all grains have been stretched to extend along loading direction. Moreover, nano-voids formation is observed on the grain boundaries, as marked with black arrows in Fig. 7a. In addition, it is observed that the atomic structure of the nanograin that highlighted with dash box in Fig. 7a has changed. A new crystalline phase is identified by coordinate number analysis, as presented in Fig. $7 \mathrm{~b}$. The representative atomic arrangements of the framed nanograin before and after deformation are displayed in Fig. 8a and Fig. 8b, respectively. In this study, both radial distribution function (RDF) and coordinate number are employed to identify the new phase. Structure and RDF analysis indicates that the distance between nearest Ca-Ca neighbors of the new phase is $3.3 \AA$ 

9. Therefore, a cutoff radius of $3.5 \AA$, which is in between $3.3 \AA$ and $3.89 \AA$, is selected to calculate the coordinate number of calcium ions. Fig. $7 \mathrm{~b}$ and Fig. $7 \mathrm{c}$ present the snapshots of deformed configurations at strains $\square=3.0 \%$ and $\square=5.0 \%$. The atoms are colored according to the coordinate number of calcium atoms with blue represents aragonite, green and cyan represent the transformed new phase and amorphous phase on grain boundaries, respectively. Comparing Fig. 7b and Fig. 7c, one can note that the new phase grows with the increase of tensile strain. In addition, the amorphous phase (cyan color) on grain boundaries dilates accordingly. Nano-voids grow up and coalesce, which eventually leads to the intergranular rupture of entire specimen.

Most importantly, the constitutive responses of the mineral-protein composite is significantly different from that of single crystalline or polycrystalline aragonite (Fig. 5a). Since fracture toughness can be determined by integrating the stress-strain curve, i.e., area beneath the curve, it can be concluded that the fracture toughness of mineral-protein composite is significantly larger than that of single or polycrystalline aragonite. Fig. 10 summaries the Young's modulus, fracture strength and toughness of the simulated computer models and experimental results. It can be noted that the overall pattern of mechanical properties of simulated samples is very similar to that of experimental materials.

To identify the deformation mechanism of mineral-protein composite that gives rise to high work-offracture, we track the atomic configurations under various strains. Fig. 11a and Fig. 11b present the atomic arrangements under strains of $\square=3 \%$ and $\square=8 \%$. It can be noted from the comparison of these two figures that, with the increase of tensile strain, nanograins are stretched to extend along loading direction. Meanwhile, the width of grain boundaries also increases, which is similar to the observation of polycrystalline aragonite under uniaxial tension. However, with the additive of protein on boundaries, the yielding of mineral-protein composite is not due to inter-granular fracture as observed in polycrystalline aragonite. Fig. 11c presents the detailed atomic arrangement of the framed region in Fig. 11b. Proteins on grain boundaries are colored according to residue types, with red and blue represent acidic and basic amino acids, and green and white represent polar/non-polar amino acids and water molecules. The circles and boxes in Fig. 11c highlight the electrostatic interaction between protein and mineral. One can recognize that the acidic (red) and basic (blue) amino acids have an obvious tendency to adhere to calcium carbonate ions, which is consistent with the observation in the work by Zhang and Chen [29], in which the electrostatic interaction was demonstrated to be responsible for the sawtooth behavior observed from AFM experiment [17]. It is well known that electrostatic interaction is more than twenty times stronger than the hydrogen bonds that initiated by water molecules or van der Waals interaction. In addition, soft organic material is well known by its high ductility. Therefore, the grain boundaries are stretched to dilate, whereas not ruptured. In contrast, as the tensile strain increases to a critical value, intra-granular fracture along [-110] direction is observed as marked by dash lines in Fig. 11c. This phenomenon can be elucidated from the aspect of energy dissipation, i.e., $E=F \times D$, where $E, F$ and $D$ represent the dissipation of energy, interactive force and displacement between atoms, respectively. Although the interactive force $F$ within aragonite nanograin is larger than that between protein and 
mineral on grain boundaries, protein exhibits significant higher resistance to plastic deformation than aragonite does (large $D$ ). Hence, with the increase of energy dissipation $E$, aragonite nano-grains fail through fracture along [-110] cleavage plane due to the low resistance to plastic deformation (small $D$ ); in comparison, proteins are extended but no obvious inter-granular fracture is observed. We believe that the strong electrostatic interaction between protein and aragonite grains, as well as the high ductility of protein, make the mineral-protein composite much tougher than pure aragonite, as shown in Fig. 5a and Fig. 10 .

\section{Conclusions}

In summary, a mineral-protein composite model is constructed through SMD to investigate the nanoscale toughening mechanism of mimic nacre tablet,. Room temperature MD simulations are then performed on single crystalline aragonite, polycrystalline aragonite and mineral-protein composite. Simulation results suggest that the pattern of the obtained stress-strain curves is in good agreement with that of experiment measurement. The work-of-fracture, which is determined by the area beneath stress-strain curve, of the mineral-protein composite is significantly larger than that of single crystalline or polycrystalline aragonite.

Deformation mechanisms such as phase transformation, dislocation motion and intra- and inter-granular failure have been identified at the atomic level, as summarized in Table 2. Single crystalline aragonite fails through dislocation nucleation and motion, as well as phase transformation. Similarly, during the deformation process of polycrystalline aragonite under tension, phase transformation is also observed in the individual nano-grains. Voids form and coalesce along grain boundaries, leading to the inter-granular failure of the entire specimen. In contrast, intra-granular cleavage is observed in the mineral-protein composite. The soft protein chains serve as strong and tough glue between nanograins. The strong electrostatic interactions between protein and aragonite nanograins, combined with the high ductility of protein are believed to be responsible for the high fracture toughness of mineral-protein composite. Our molecular dynamics study provides insights of the toughening mechanism at the molecular level and paves a way to inspire new ideas for fabricating novel tough materials.

\section{Acknowledgements}

This work was supported by National Science Foundation under Award Numbers CMMI-0824688 and CMMI-0855795. Simulations were performed at the High Performance Computing Center at the University of Florida.

\section{Author contributions}

Y.C. conceived the original idea. Y.C. and N.Z. designed the research. N.Z., S.Y., and Y.H. performed atomistic simulation, analyzed the data and prepared the figures. N.Z. wrote the manuscript. Y.C. and L.X. advised on the simulation and the interpretation of the results.

\section{Conflict of interest:}

The authors declare no competing financial interest. 


\section{References}

1. Meyers, M.A., et al., Biological materials: a materials science approach. Journal of the Mechanical behavior of biomedical materials, 2011. 4(5): p. 626-657.

2. Menig, R., et al., Quasi-static and dynamic mechanical response of Haliotis rufescens (abalone) shells. Acta Materialia, 2000. 48(9): p. 2383-2398.

3. Currey, J., Mechanical properties of mother of pearl in tension. Proceedings of the Royal Society of London. Series B. Biological Sciences, 1977. 196(1125): p. 443-463.

4. Jackson, A., J. Vincent, and R. Turner, The mechanical design of nacre. Proceedings of the Royal Society of London B: Biological Sciences, 1988. 234(1277): p. 415-440.

5. Jackson, A., J. Vincent, and R. Turner, A physical model of nacre. Composites Science and Technology, 1989. 36(3): p. 255-266.

6. Sarikaya, M., et al. Mechanical property-microstructural relationships in abalone shell. in MRS Proceedings. 1989: Cambridge Univ Press.

7. Kotha, S., Y. Li, and N. Guzelsu, Micromechanical model of nacre tested in tension. Journal of materials science, 2001. 36(8).

8. Wang, R., et al., Deformation mechanisms in nacre. Journal of Materials Research, 2001. 16(09): p. 2485-2493.

9. Song, F. and Y. Bai, Effects of nanostructures on the fracture strength of the interfaces in nacre. Journal of Materials Research, 2003. 18(08): p. 1741-1744.

10. Barthelat, F., et al., On the mechanics of mother-of-pearl: a key feature in the material hierarchical structure. Journal of the Mechanics and Physics of Solids, 2007. 55(2): p. 306-337.

11. Katti, K.S., et al., Platelet interlocks are the key to toughness and strength in nacre. Journal of Materials Research, 2005. 20(05): p. 1097-1100.

12. Meyers, M.A., et al., Mechanical strength of abalone nacre: role of the soft organic layer. Journal of the Mechanical behavior of biomedical materials, 2008. 1(1): p. 76-85.

13. Barthelat, F., et al., Mechanical properties of nacre constituents and their impact on mechanical performance. Journal of Materials Research, 2006. 21(08): p. 1977-1986.

14. Meyers, M.A., et al., Biological materials: structure and mechanical properties. Progress in Materials Science, 2008. 53(1): p. 1-206.

15. Verma, D., K. Katti, and D. Katti, Photoacoustic FTIR spectroscopic study of undisturbed nacre from red abalone. Spectrochimica Acta Part A: Molecular and Biomolecular Spectroscopy, 2006. 64(4): p. 1051-1057.

16. Katti, K.S., B. Mohanty, and D.R. Katti, Biomimetic lessons learnt from nacre. 2010: INTECH Open Access Publisher.

17. Smith, B.L., et al., Molecular mechanistic origin of the toughness of natural adhesives, fibres and composites. Nature, 1999. 399(6738): p. 761-763.

18. $\mathrm{Xu}, \mathrm{Z} . \mathrm{H}$. and X. Li, Deformation strengthening of biopolymer in nacre. Advanced Functional Materials, 2011. 21(20): p. 3883-3888.

19. Li, X., et al., Nanoscale structural and mechanical characterization of a natural nanocomposite material: the shell of red abalone. Nano Letters, 2004. 4(4): p. 613-617.

20. Sun, J. and B. Bhushan, Hierarchical structure and mechanical properties of nacre: a review. Rsc Advances, 2012. 2(20): p. 7617-7632.

21. Shen, X., et al., Molecular cloning and characterization of lustrin A, a matrix protein from shell and pearl nacre of Haliotis rufescens. Journal of Biological Chemistry, 1997. 272(51): p. 3247232481. 

from the shell of the mollusc Haliotis laevigata. Biochemical and biophysical research communications, 2000. 267(1): p. 17-21.

23. Weiss, I.M., et al., Perlustrin, a Haliotis laevigata (abalone) nacre protein, is homologous to the insulin-like growth factor binding protein $\mathrm{N}$-terminal module of vertebrates. Biochemical and biophysical research communications, 2001. 285(2): p. 244-249.

24. Treccani, L., et al., Perlwapin, an abalone nacre protein with three four-disulfide core (whey acidic protein) domains, inhibits the growth of calcium carbonate crystals. Biophysical journal, 2006. 91(7): p. 2601-2608.

25. Li, X., Z.-H. Xu, and R. Wang, In situ observation of nanograin rotation and deformation in nacre. Nano Letters, 2006. 6(10): p. 2301-2304.

26. Ortiz, C. and M.C. Boyce, Bioinspired structural materials. Science, 2008. 319(5866): p. 10531054

27. Zhang, N. and Y. Chen, Molecular origin of the sawtooth behavior and the toughness of nacre. Materials Science and Engineering: C, 2012. 32(6): p. 1542-1547.

28. Zhang, N., et al., Deformation mechanisms in silicon nanoparticles. Journal of Applied Physics, 2011. 109(6): p. 063534.

29. Zhang, N. and Y. Chen, Nanoscale plastic deformation mechanism in single crystal aragonite. Journal of materials science, 2013. 48(2): p. 785-796.

30. Mann, K., et al., The amino - acid sequence of the abalone (Haliotis laevigata) nacre protein perlucin. European Journal of Biochemistry, 2000. 267(16): p. 5257-5264.

31. Cornell, W.D., et al., A second generation force field for the simulation of proteins, nucleic acids, and organic molecules. Journal of the American Chemical Society, 1995. 117(19): p. 5179-5197.

32. Plimpton, S., Fast parallel algorithms for short-range molecular dynamics. Journal of computational physics, 1995. 117(1): p. 1-19.

33. Jorgensen, W.L., et al., Comparison of simple potential functions for simulating liquid water. The Journal of chemical physics, 1983. 79(2): p. 926-935.

34. Dove, M.T., et al., A new interatomic potential model for calcite: applications to lattice dynamics studies, phase transition, and isotope fractionation. The American mineralogist, 1992. 77(3-4): p. 244-250.

35. Verlet, L., Computer" experiments" on classical fluids. I. Thermodynamical properties of LennardJones molecules. Physical review, 1967. 159(1): p. 98.

36. van Gunsteren, W.F. and H.J. Berendsen, Computer simulation of molecular dynamics: Methodology, applications, and perspectives in chemistry. Angewandte Chemie International Edition in English, 1990. 29(9): p. 992-1023.

37. Woodcock, L.-V., Isothermal molecular dynamics calculations for liquid salts. Chemical Physics Letters, 1971. 10(3): p. 257-261.

38. Nosé, S., A unified formulation of the constant temperature molecular dynamics methods. The Journal of chemical physics, 1984. 81(1): p. 511-519. 


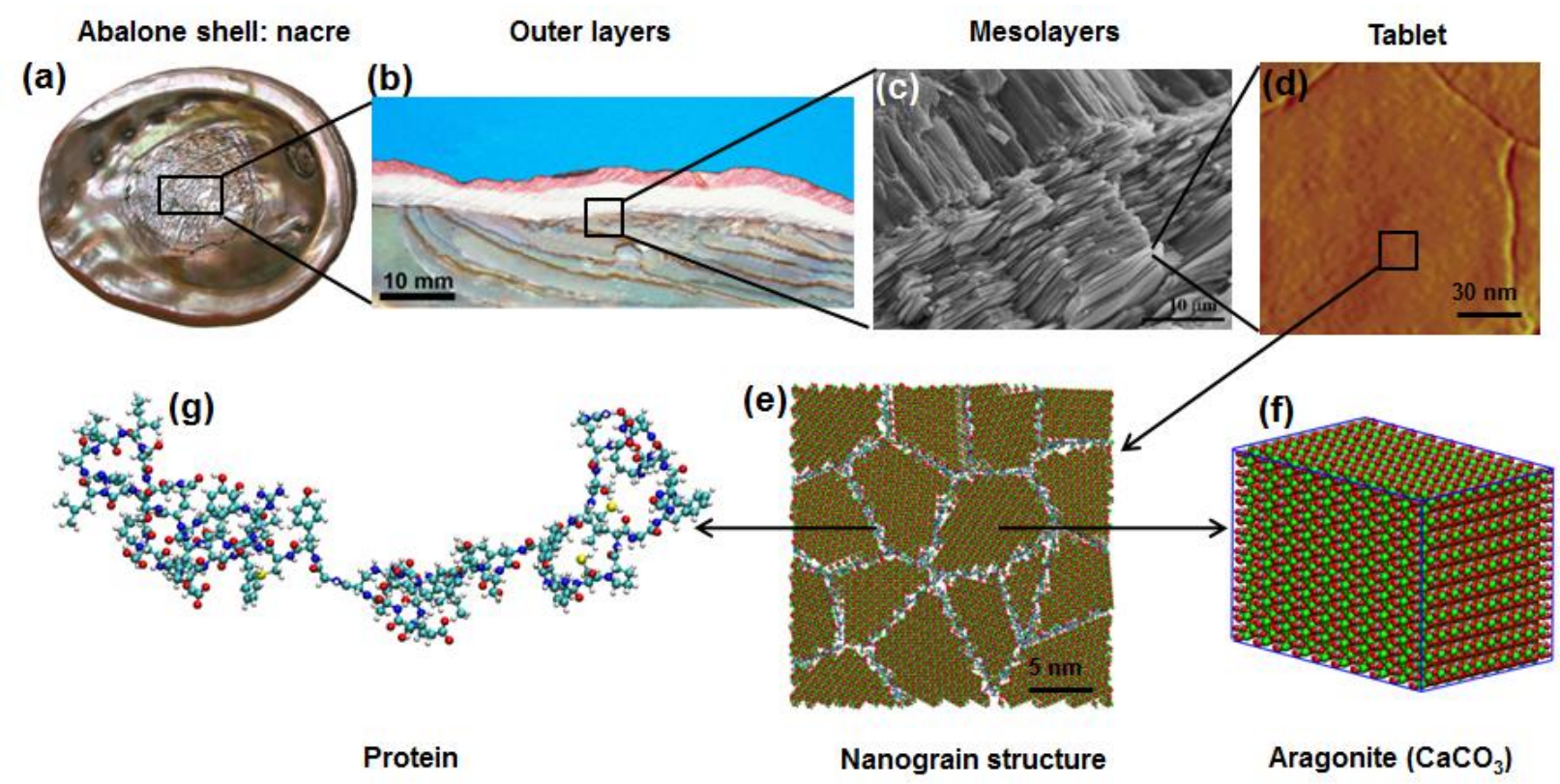

Fig. 1 - Hierarchical structure of the red abalone nacre. (a) View of the inner nacreous layer of red abalone shell [1]. (b) Cross section view of layers [1]. (c) SEM image of fracture surface of mesolayers [1]. (d) AFM image of tablet [1]. (e) Atomic configuration of mineral-protein composite. (f) The 3-D structure of aragonite within nanograins. (g) Protein on nanograin boundaries.
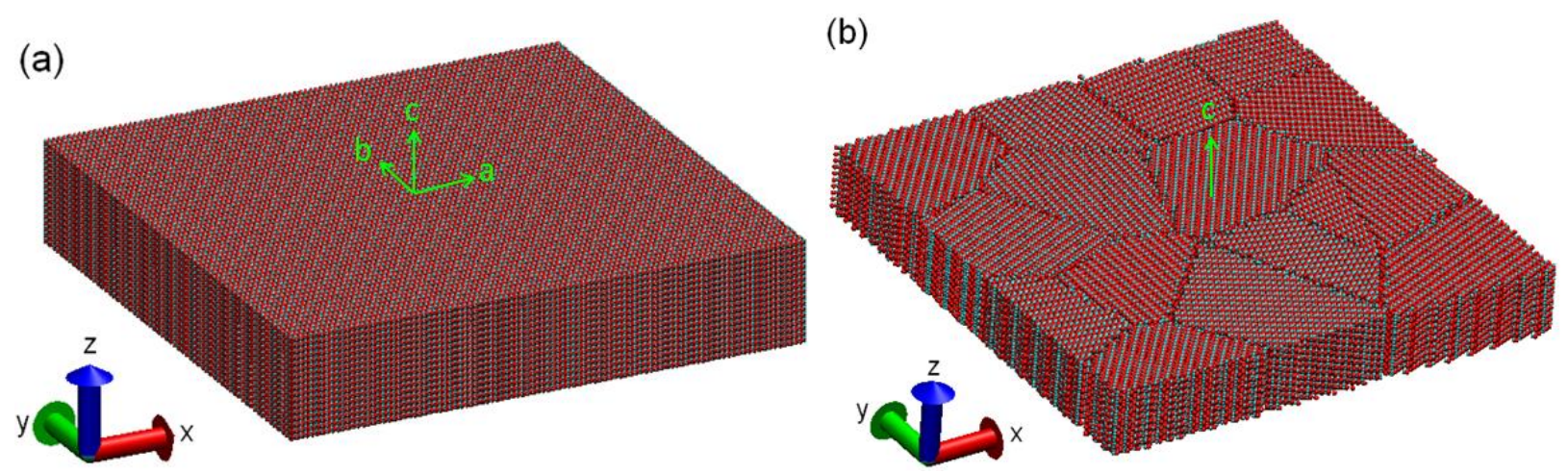

Fig. 2 - Initial atomic configurations of (a) single crystalline aragonite and (b) polycrystalline aragonite. The crystallographic orientations: a-, b- and c-axis are denoted. 


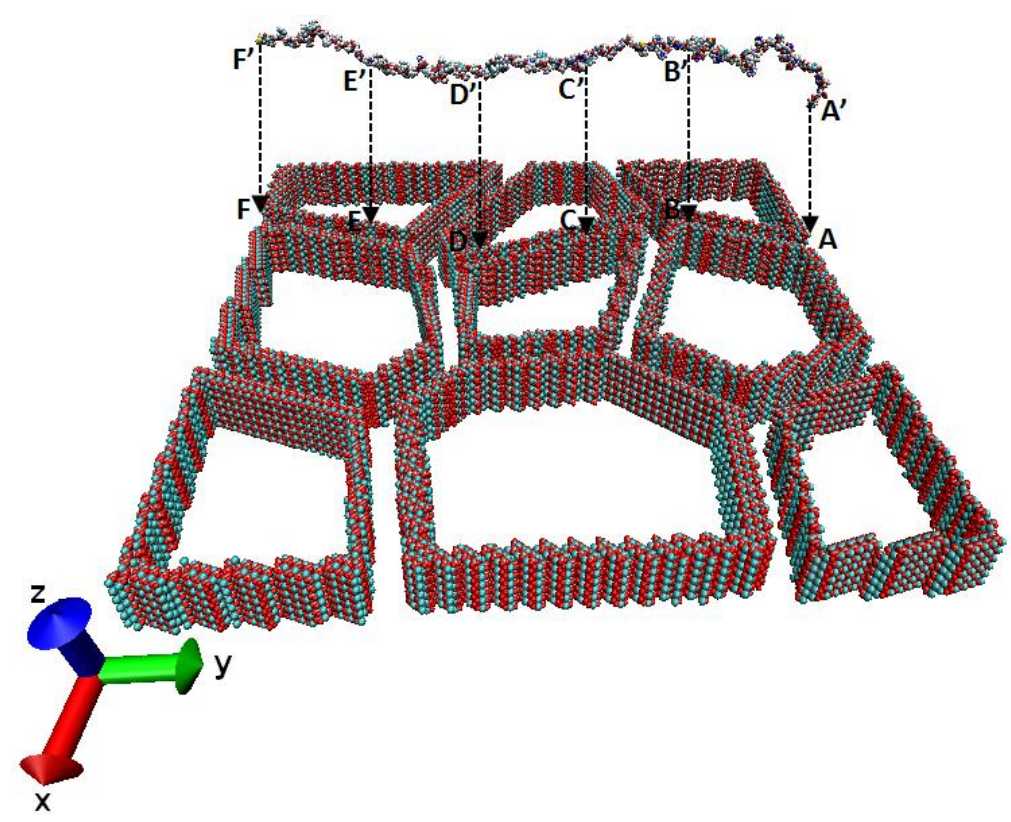

Fig. 3 - Schematic representation of the initial computer model, in which predefined reference points along grain boundaries and protein chain are marked as A, B..., F and A', B'..., F', respectively.
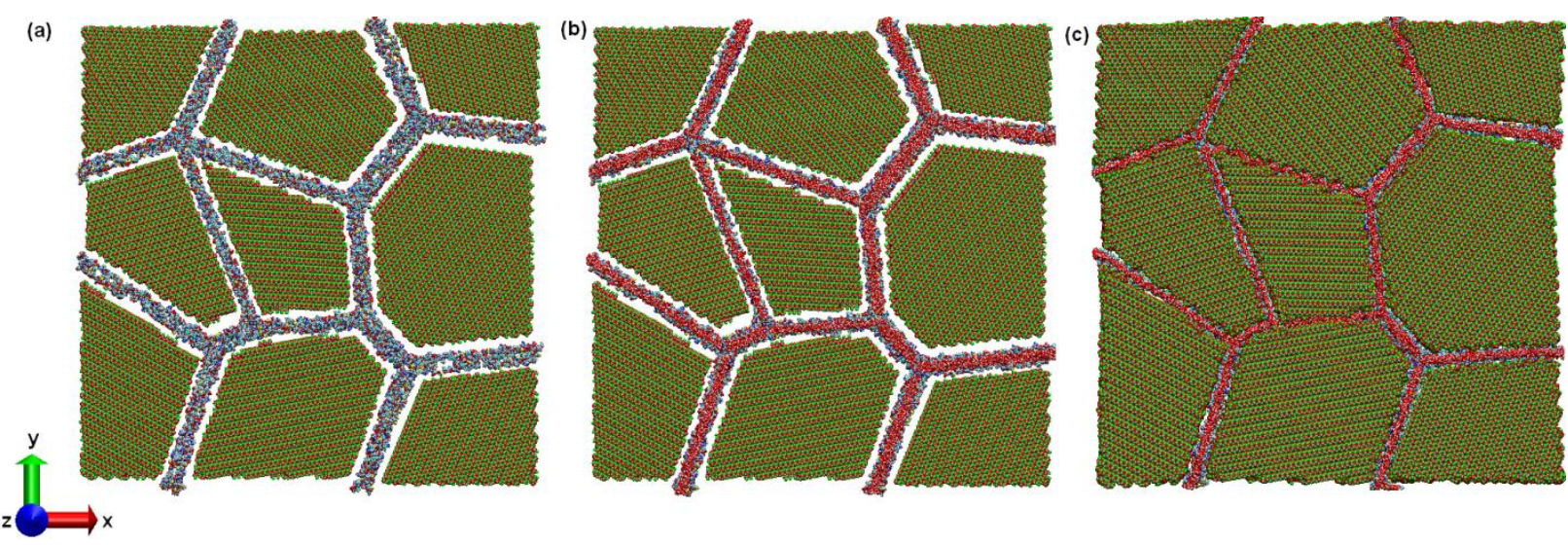

Fig. 4 - Top views of mineral-protein composite model. (a) Protein chains are stretched into grain boundaries. (b) Water molecules are randomly scattered on protein surfaces. (c) Spacing between protein and mineral is eliminated by squeezing the initial model. 

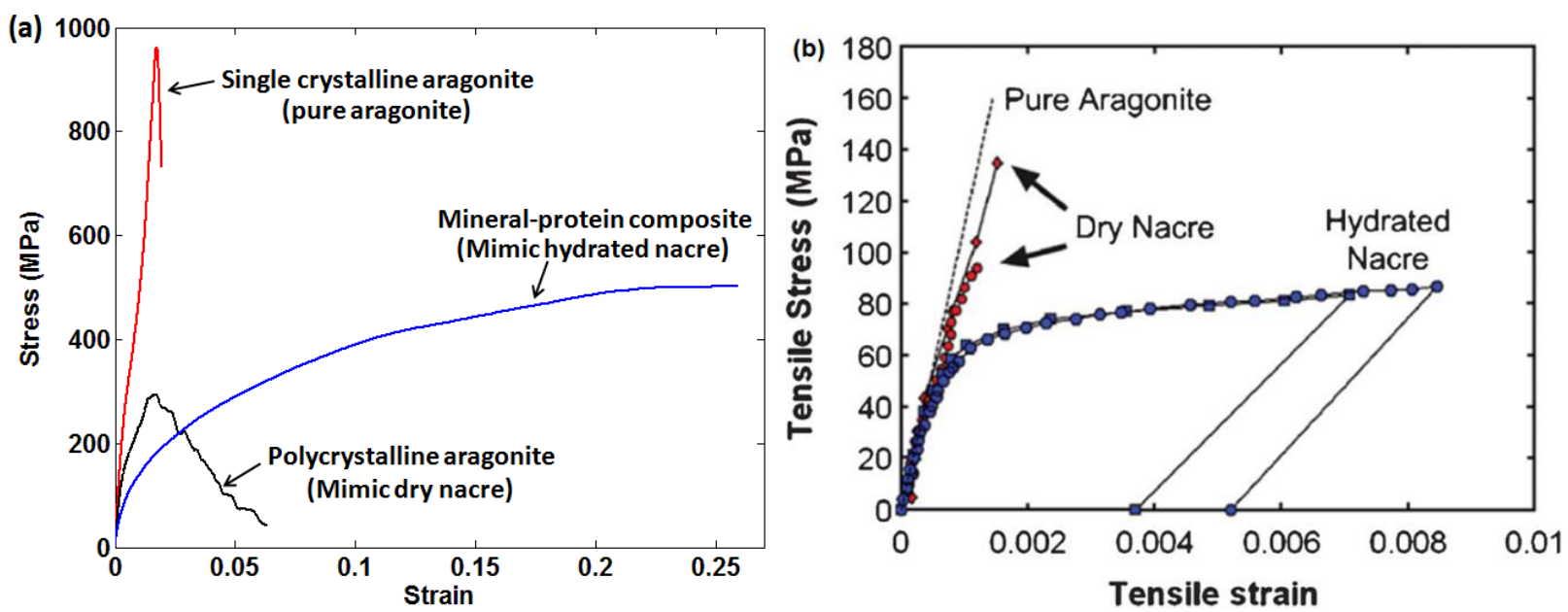

Fig. 5 - (a) Stress-strain curves for single crystalline aragonite, polycrystalline aragonite and mineralprotein composite under uniaxial tension obtained from current MD simulation. (b) stress-strain relations of nacre measured from experiment [20].
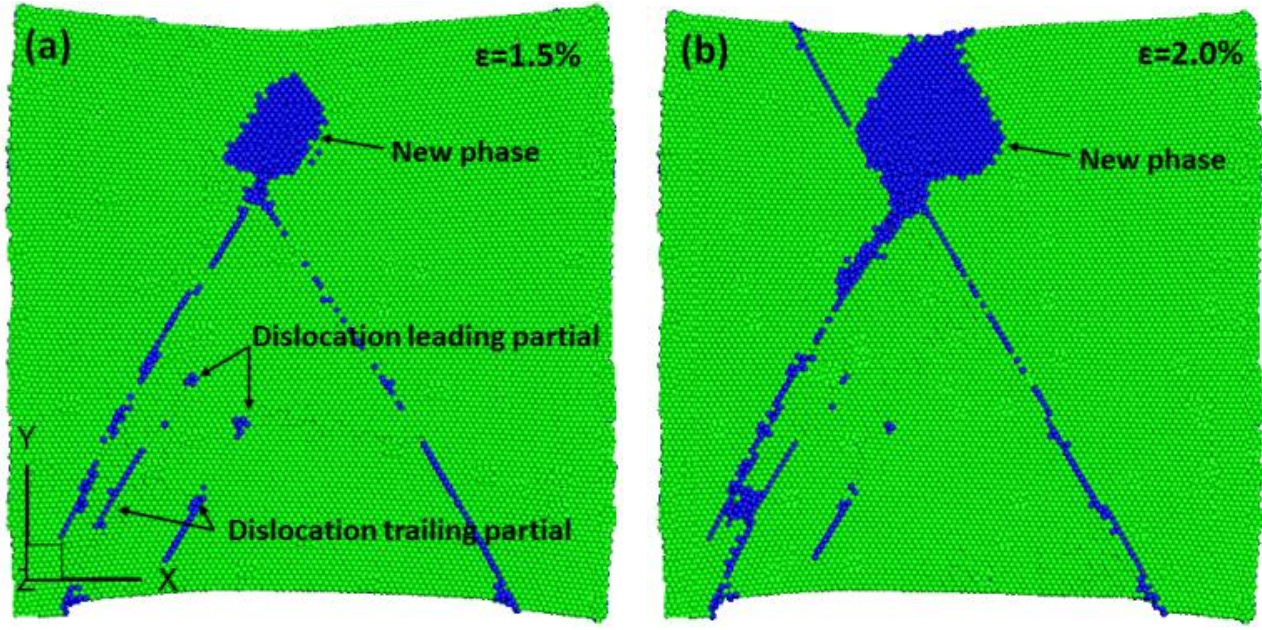

Fig. 6 - Snapshots of single crystalline aragonite under uniaxial tension at strains (a) $\square=1.5 \%$ and (b) $\square=2.0 \%$. The atoms are colored by coordinate number with green color represents original aragonite; blue color represents new phase and dislocation lines. 

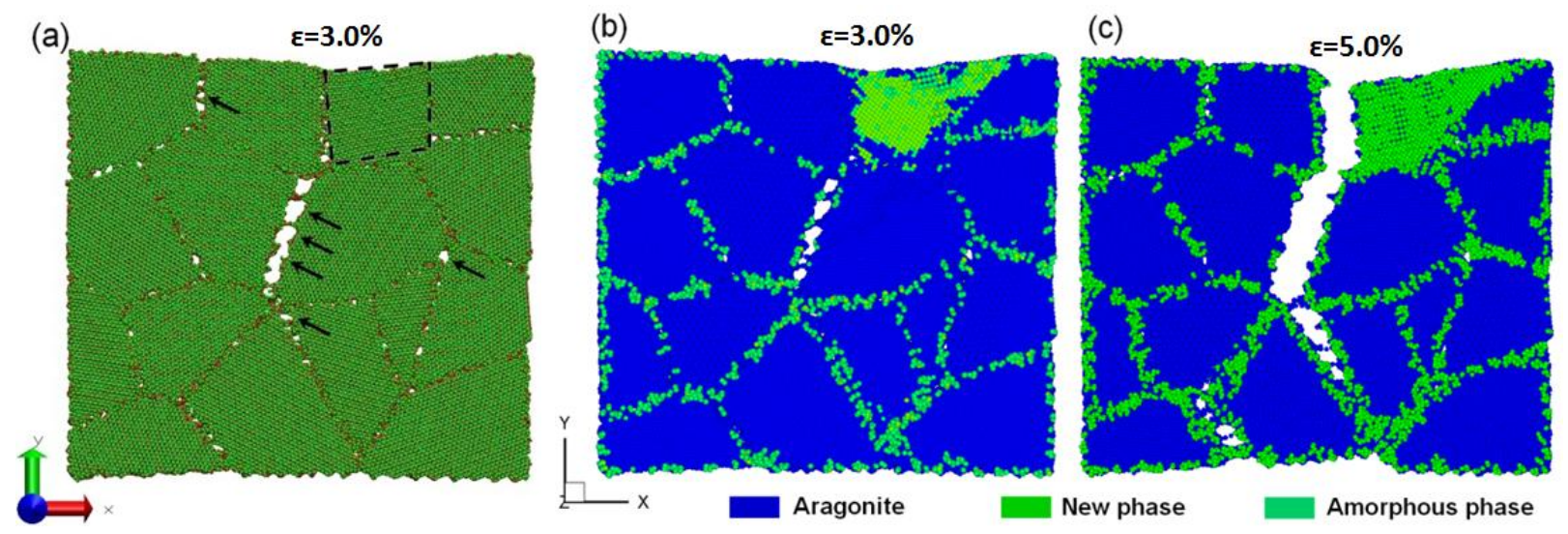

Fig. 7 - Snapshots of deformed polycrystalline aragonite at strains (a-b) $\square=3.0 \%$ and (c) $\square=5.0 \%$. The atoms in (b) and (c) are colored by coordinate number.

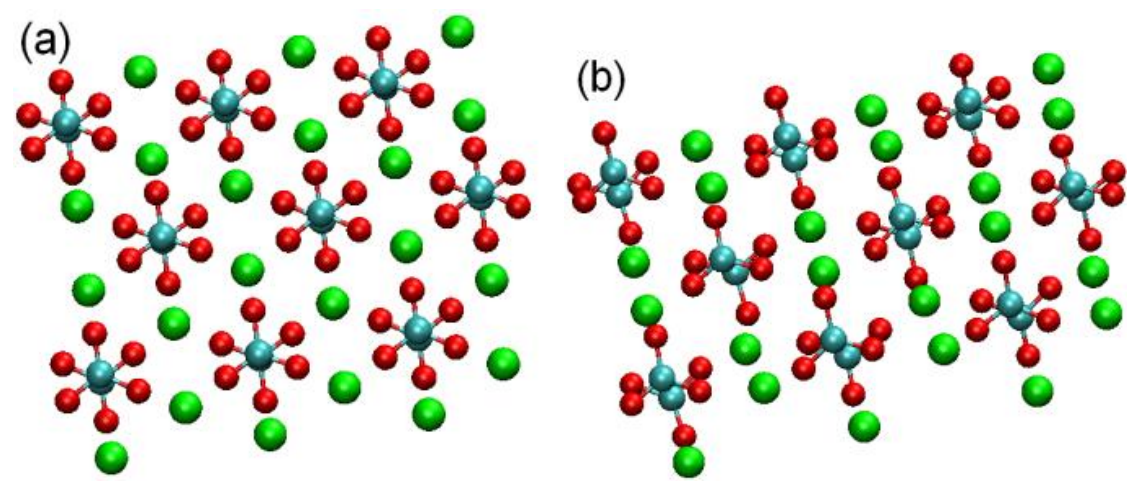

Fig. 8 - Comparison of atomic arrangements of (a) aragonite and (b) new phase.

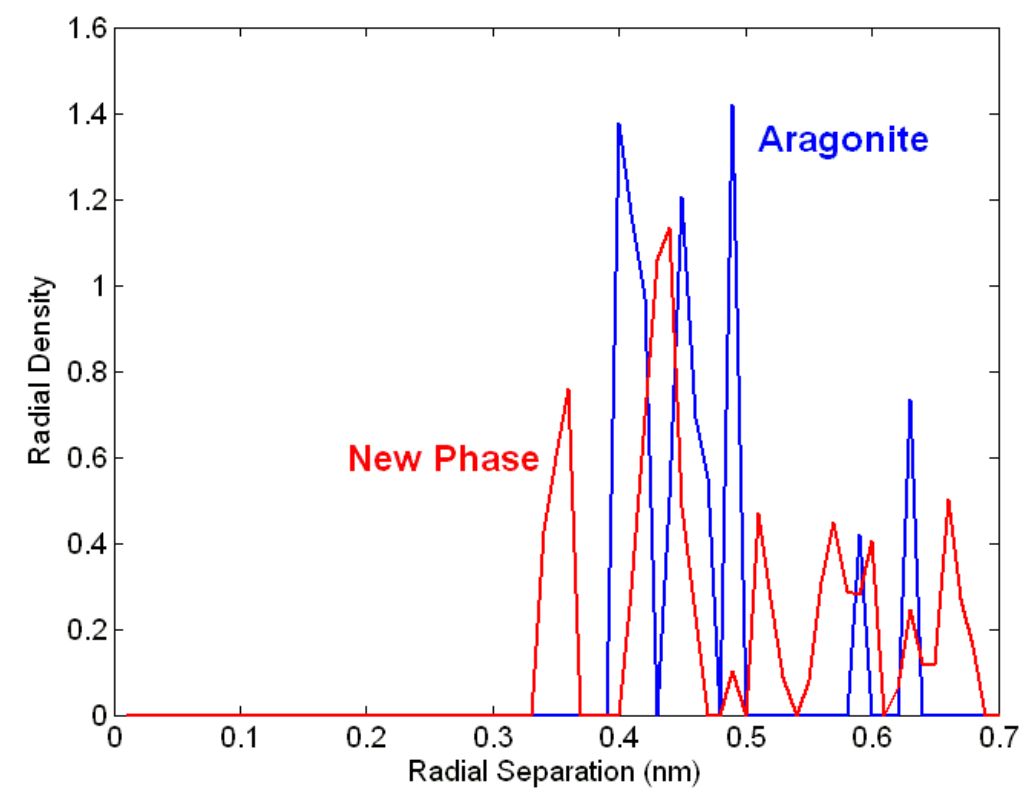


Fig. 9 - Radial distribution function (RDF) of calcium-calcium in aragonite (blue lines) and new phase (red lines).

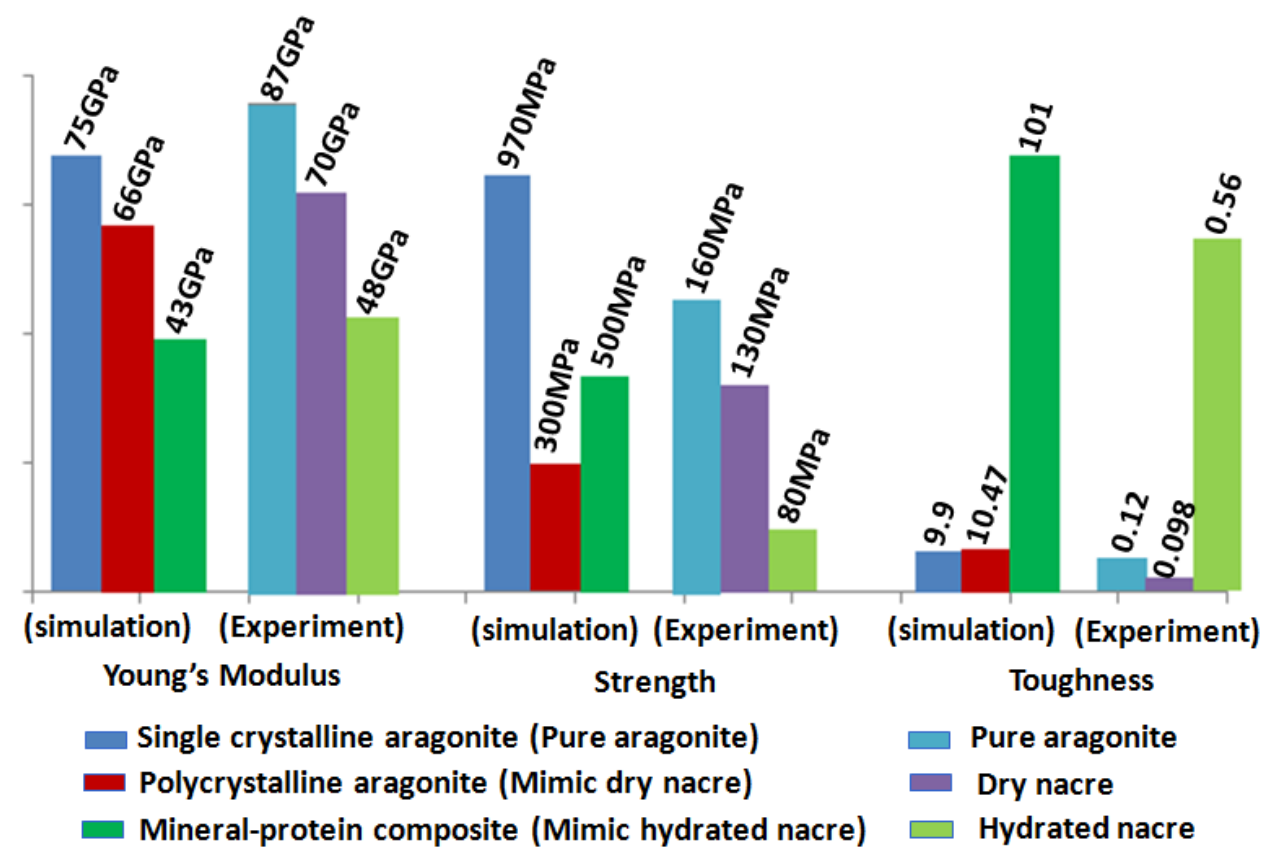

Fig. 10 - Comparison of Young's modulus, fracture strength and toughness among single crystalline aragonite, polycrystalline aragonite and mineral-protein composite obtained from present computational work, as well as the measurement from experiment. Fracture toughness is obtained by calculating the area beneath stress-strain curve.
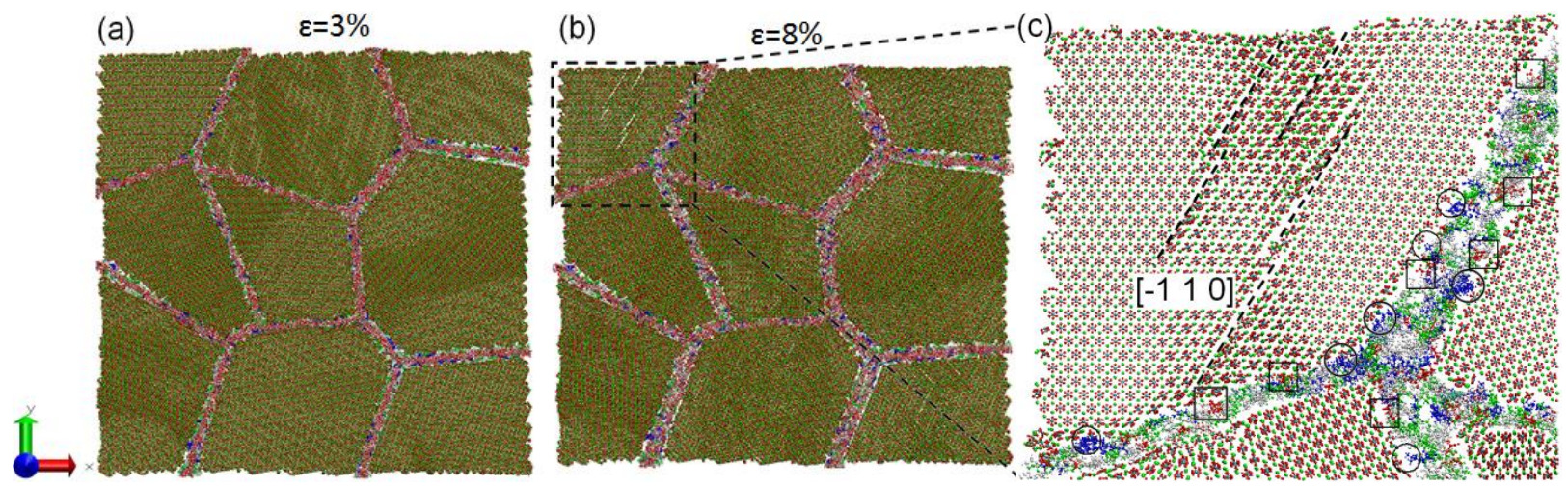

Fig. 11 - Atomic arrangements of mineral-protein composite at strains (a) $\square=3.0 \%$ and (b) $\square=8.0 \%$. (c) Zoomed in atomic configuration of the dash boxed region in (b).

Table 1. Force field parameters for crystalline aragonite. 


\begin{tabular}{lll}
\hline Interaction & $A(\mathrm{eV})$ & $\rho(\AA)$ \\
$\mathrm{Ca}-\mathrm{O}$ & 3943.5977 & 0251570 \\
$\mathrm{O}-\mathrm{O}$ & 2879.1262 & 0.252525 \\
$\mathrm{C}-\mathrm{O}$ & $1.7411309 \times 10^{13}$ & 0.03873 \\
\hline $\mathrm{Ca}$ charge $=+1.64203 \mathrm{e}$ & $\mathrm{k}_{\theta}(\mathrm{O}-\mathrm{C}-\mathrm{O})=3.69441 \mathrm{eV} \mathrm{rad}^{-2}$ & \\
$\mathrm{O}$ charge $=-0.894293 \mathrm{e}$ & $\mathrm{k}_{\mathrm{t}}(\mathrm{O}-\mathrm{C}-\mathrm{O})=0.125125 \mathrm{eV}$ & \\
$\mathrm{C}$ charge $=+1.04085 \mathrm{e}$ & & \\
\hline
\end{tabular}

Table 2. A summary of deformation mechanisms.

\section{Single crystalline aragonite Polycrystalline aragonite Mineral-protein composite}

$\begin{array}{llll}\text { Deformation } & \text { (1) Phase transformation } & \text { (1) Phase transformation } & \text { (1) Extension of protein } \\ \text { mechanisms } & \text { (2) Dislocation motion } & \text { (2) Inter-granular fracture } & \text { (2) Intra-granular fracture }\end{array}$

